\title{
COMMENT
}

Check for updates

\section{Community-driven online initiatives have reshaped scientific engagement}

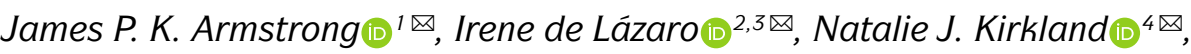 \\ Wilson Poon $\mathbb{1}^{5,6 凶}$ and Shrey Sindhwani(10 ${ }^{7 凶}$
}

Scientists have reacted to COVID-19 restrictions by organizing virtual seminars and journal clubs to maintain engagement. We reflect on our experiences and lessons learned from organizing such initiatives and highlight how, far from being temporary substitutes of in-person counterparts, they can help foster more diverse, inclusive and environmentally friendly scientific exchange.

Science thrives on collaboration, connectivity and knowledge exchange ${ }^{1}$. Research groups gather for journal clubs to share new developments in their field, departments congregate to see guest speakers convey their latest findings, and larger communities assemble at conferences to present, learn, discuss, debate and build valuable networks of collaborators. Such activities were abruptly halted by the COVID-19 pandemic, which triggered lockdowns, travel restrictions and temporary closures at many universities and scientific institutions ${ }^{2}$. This unique situation created a profound transition in scientific engagement, with a rapid proliferation of online conferences and meetings, often staged by professional companies and established scientific societies ${ }^{3}$. However, the pandemic has also led to the rise of virtual events organized and chaired on a voluntary basis by independent scientists, often trainees with little or no prior experience of event management. Yet these community-driven initiatives, unburdened by corporate shackles and empowered by technology and social media, have been a successful and adaptive forum for online scientific engagement.

We are five early-career scientists, who have spearheaded three popular online initiatives oriented to fill different gaps in knowledge exchange caused by the pandemic. Irene de Lázaro co-organizes and chairs Topics in Bioengineering, James Armstrong and Natalie Kirkland have been the principal chairs of Virtual Seminars in Biomedical Sciences, and Wilson Poon and Shrey Sindhwani lead the Nanomedicine Journal Club. We reflect on our experiences in running these events and the aspects that we would like to retain when academic life recovers its full normalcy.

\section{Topics in Bioengineering}

The Topics in Bioengineering trainee-led seminar series has been running at the Harvard John A. Paulson School of Engineering and Applied Sciences since 2014. Before COVID-19, established investigators primarily from US-based institutions - would visit every fortnight and spend the day on campus to deliver a seminar and meet with trainees and faculty. When it became evident that the disruptions caused by the pandemic would last for months or years, rather than weeks, we decided to migrate to an 'all-welcome' virtual format. This has broadened the event, allowing us to host more speakers based outside of North America (FIG. 1a) and attract more than 1,000 registrants from at least 39 different countries (FIG. 1 b). The virtual format democratizes access to what used to be an exclusively departmental activity, and a more scientifically diverse audience has allowed us to broaden the scope of bioengineering talks when previously we might have feared low attendance. The only downside has been the loss of in-person interactions, which has been partially addressed by including a post-seminar 'virtual happy hour' for departmental trainees to chat with the speakers in a more casual environment. However, this does not compare to the day-long event and one-on-one meetings of pre-pandemic times. For this reason, I am excited to welcome speakers back on campus once it is safe, but will advocate for a hybrid model that grants the option to visit virtually and enables the online broadcast of in-person events to maintain global access.

\section{Virtual Seminars in Biomedical Sciences}

The Virtual Seminars in Biomedical Sciences online seminar series was established in May 2020 and at its peak ran four times a month. The stated remit was to "amplify the voices of graduate students and postdoctoral researchers", an academic demographic that has been disproportionately affected by event cancellations ${ }^{4}$. We were struck by the willingness of speakers and attendees to engage with this format, actively participating in seminar discussions and promoting the series across social media. This engagement has built a fantastic new community, predominantly composed of early-career researchers, which has even led to new scientific collaborations. An unforeseen occurrence, sparked by the Black Lives Matter protests in 2020, was the discussion about racial disparities in health research and academia, highlighted by several presenters. 
a Topics in Bioengineering - speakers

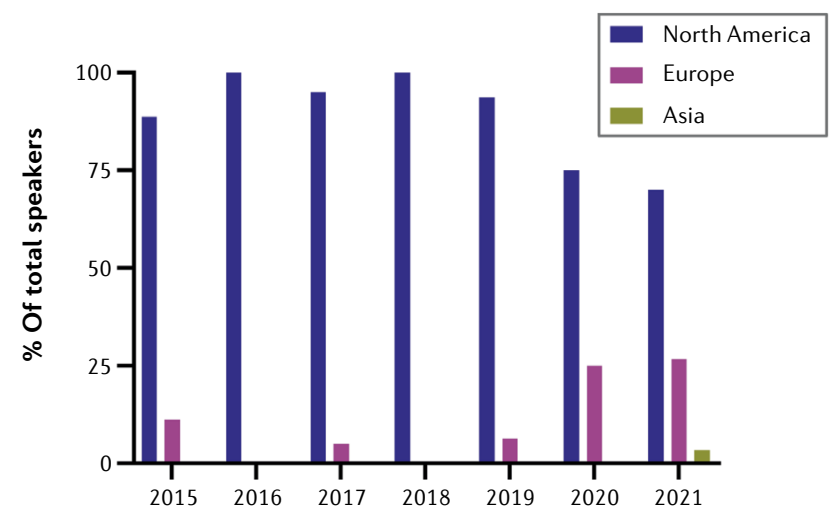

C Virtual Seminars in Biomedical Science - registrants

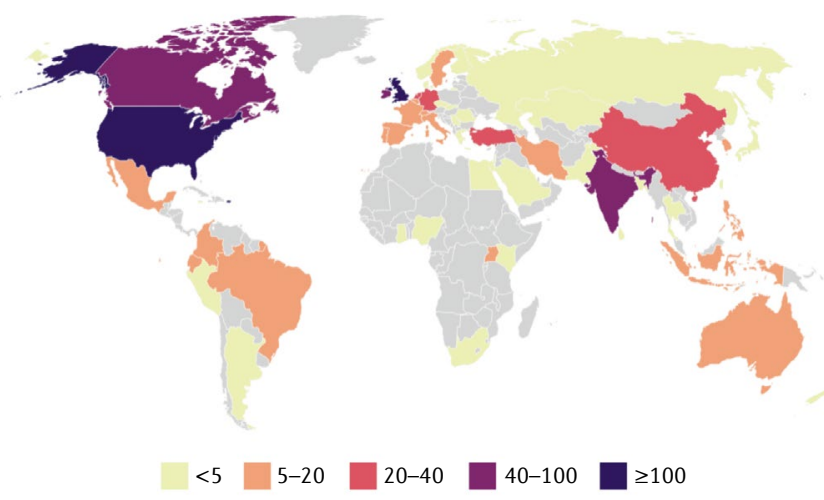

Created with Datawrapper

Fig. 1 | The global reach of community-driven online initiatives that have emerged during the COVID-19 pandemic. a | Topics in Bioengineering speakers from institutions based in North America, Europe and Asia when held in-person (2015-2019) and virtually (2020-2021). b-d | The worldwide distribution of registrants for each of the three b Topics in Bioengineering - registrants

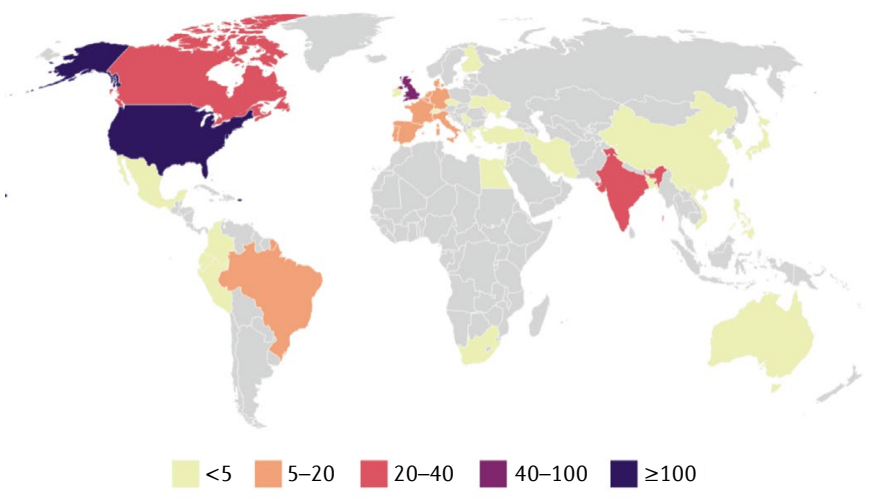

Created with Datawrapper

d Nanomedicine Journal Club - registrants

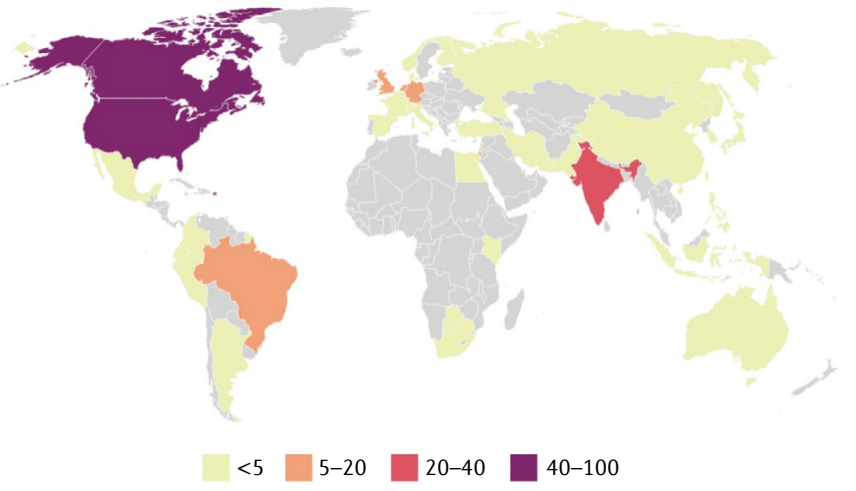

Created with Datawrapper

initiatives, determined by the institutional addresses used at registration. Geographical data from participants that registered with non-institutional e-mail addresses could not be determined and were therefore not included in the analysis. The key shows the number range of registered individuals, as of August 2021. World map figures plotted using Datawrapper.
As an independent initiative, we were able to accommodate this change in scope and fully support these discussions.

Furthermore, we strongly advocate free-to-access, accessible science and are proud to have attracted more than 1,000 registrants across six continents (FIG. 1c). We have welcomed attendees not affiliated with any scientific institution, with speakers frequently inviting friends and family to watch their talks. This has created an openness about the events, and attendees have commented on how it is less intimidating to ask questions online than in-person. We regularly attracted more than 100 attendees per seminar in the early series but, following the easing of restrictions and reopening of laboratories, it has been challenging to maintain the high attendances required for effective engagement. Therefore, after 84 seminars, we concluded this series in September 2021.

\section{Nanomedicine Journal Club}

This online journal club started in March 2020 and was initially intended for a small group of affiliated laboratories. However, with encouragement from the online nanomedicine community, it evolved into a public series that continues to run twice a month and gives trainees an opportunity to present their own work or studies conducted by others. Three goals were established at the outset. First, to allow speakers to select seminal papers to re-evaluate evidence of key concepts that have shaped the field and address fundamental gaps in knowledge. Second, to expose our audience to the latest work involving technologies and techniques that are not commonly used in nanomedicine but could add significant value. With the excitement around nanoparticle-based COVID-19 vaccines, we included studies on vaccine development, immunological assays, genetic engineering and molecular biology techniques. We hope that this scope broadens our understanding of future nanomedicine studies and enables researchers to effectively communicate with the public. Third, we want to have rigorous discussion after each presentation, delving deep into the results and methodology that support the claims.

Traditional journal clubs comprising one or two research groups can often become entrenched in similar viewpoints. The broad knowledge base and dynamic participation of individuals from different laboratories (FIG. 1d) has enriched our discussions. To date, the journal club has featured more than 35 presenters from across North America, Europe and Asia, and we will continue 
as long as there is interest and active participation from the community.

\section{Lessons learned and moving forward}

Community-driven online initiatives have played an important role in maintaining scientific engagement during the COVID-19 pandemic. They have enabled scientists, especially early-career researchers, to build networks that are valuable for career progression, to find jobs and to learn about different research laboratories. More generally, they have provided a sense of community in a time when many have faced isolation ${ }^{5}$. These initiatives have successfully adopted the regularity and format of traditionally small events (for example, research group journal clubs and departmental seminars), while attracting global audiences. This geographical diversity has enriched scientific discussions, and online engagement has removed many of the barriers to participation for those with caring responsibilities, disabilities or availability and financial restrictions ${ }^{6}$. Indeed, it may be argued that such initiatives have not only maintained engagement but broadened engagement. Notably, the online format enables this increase in breadth while minimizing travel. The resulting, ameliorating effect upon climate change is another considerable benefit ${ }^{7}$.

We hope that such initiatives are not seen as temporary substitutes, in place only for the duration of the pandemic. Instead, we believe that they can reshape scientific engagement to make it a more diverse, inclusive and environmentally friendly activity. However, the increasing offer of online events, video-conferencing fatigue and the progressive return to normal working patterns can pose challenges to participation. Thus, a key question is how these initiatives can evolve to provide continued engagement beyond the pandemic. Events that existed in-person before COVID-19 might benefit from a hybrid model that allows presenters and attendees to participate either in-person or virtually. In all cases, tuning the frequency of events may help to maintain a regular, but not exhausted, audience. We also advocate the use of social media to reach a large, diverse audience, outside of institutional or society mailing lists. Finally, financial support from funders would greatly aid event logistics, in particular, by enabling the use of licensed webinar platforms that provide full cybersecurity options.

Overall, we are delighted with the engagement that community-driven online events have fostered during the COVID-19 pandemic and hope that the steps outlined above can help to incorporate many of its positive aspects in the new normal of scientific exchange.

1. Ellemers, N. Science as collaborative knowledge generation. Br. J. Soc. Psychol. 60, 1-28 (2021)

2. Viglione, G. A year without conferences? How the coronavirus pandemic could change research. Nature 579, 327-328 (2020).

3. Woolston, C. Learning to love virtual conferences in the coronavirus era. Nature 582, 135-136 (2020).

4. Weissgerber, T. et al. Mitigating the impact of conference and travel cancellations on researchers' futures. eLife 9, e57032 (2020).

5. Sharma, A. Finding community during a pandemic. Science $\mathbf{3 6 8 ,}$ 206 (2020).

6. Sarabipour, S. Virtual conferences raise standards for accessibility and interactions. eLife 9, e62668 (2020).

7. Klöwer, M., Hopkins, D., Allen, M. \& Higham, J. An analysis of ways to decarbonize conference travel after COVID-19. Nature 583, 356-359 (2020).

\section{Acknowledgements}

I.d.L. acknowledges the Topics in Bioengineering organization team at the Harvard John A. Paulson School of Engineering and Applied Sciences for their contributions running the seminar during the COVID-19 pandemic, as well as invited speakers for their time and enthusiastic participation. J.P.K.A. and N.J.K. acknowledge B. Aguado and S. Quackenbush, who have helped to organize and co-chair several Virtual Seminars in Biomedical Science, as well as the speakers that have contributed to the success of the series. W.P. and S.S. wish to acknowledge all presenters and journal club attendees for their participation and continued support.

\section{Competing interests}

The authors declare no competing interests.

Publisher's note

Springer Nature remains neutral with regard to jurisdictional claims in published maps and institutional affiliations.

\section{RELATED LINKS}

Nanomedicine Journal Club: https://tinyurl.com/NanoJournalClub Topics in Bioengineering: https://tib.seas.harvard.edu/

Virtual Seminars in Biomedical Sciences: https://www.youtube.com/ channel/UCsmlV5qnnBqRuzgbZ1IFvDQ/about?view_as=subscriber 